\title{
Kidney-lung crosstalk during SARS-CoV-2 infection: In silico hypothesis-generating method for COVID- 19 models
}

\section{Dmitry N Grigoryev ( $\nabla$ gri@uchicago.edu )}

University of Chicago Biological Sciences Division: University of Chicago Division of the Biological

Sciences https://orcid.org/0000-0002-1849-1763

Hamid Rabb

Johns Hopkins Medical Institutions: Johns Hopkins Medicine

Research article

Keywords: acute kidney injury, lung cross talk, COVID receptors ACE2

Posted Date: June 18th, 2021

DOI: https://doi.org/10.21203/rs.3.rs-629422/v1

License: (c) (i) This work is licensed under a Creative Commons Attribution 4.0 International License.

Read Full License 
Kidney-lung crosstalk during SARS-CoV-2 infection: In silico hypothesis-generating method for COVID-19 models.

Dmitry N. Grigoryev ${ }^{1 *} \&$ Hamid Rabb ${ }^{2}$

${ }^{1}$ University of Chicago Division of Biological Sciences, Chicago, IL, ${ }^{2}$ Johns Hopkins Medicine, Baltimore, MD

Short title: AKI injury COVID receptors

*corresponding author

Dmitry N Grigoryev, MD, PhD

Center for Translational Data Science

5454 South Shore Drive

Suite 2A/B

Chicago, IL 60615

Email: gri@uchicago.edu

Phone: +1 6106449260

Key words: acute kidney injury, lung cross talk, COVID receptors ACE2 


\section{Abstract \\ Background}

Publicly available genomics datasets have been growing drastically during the past decades. Although most of these datasets were initially generated to answer a pre-defined scientific question, their repurposing became useful when new challenges such as COVID-19 arise. While the establishment and use of experimental models of COVID-19 are in progress, the potential hypotheses for mechanisms of onset and progression of COVID-19 can be generated by using in silico analysis of known COVID-19 conditions and SARS-CoV-2 targets.

\section{Methods}

Selecting condition: COVID-19 infection leads to acute respiratory distress syndrome (ARDS) and acute kidney injury (AKI). There is increasing data demonstrating mechanistic links between AKI and ARDS.

Selecting targets: SARS-CoV-2 uses angiotensin-converting enzyme 2 (ACE2) and transmembrane protease, serine 2 (TMPRSS2) for cell entry. We hypothesized that modeling AKI and ARDS would lead to changes in kidney and lung ACE2 and TMPRSS2. We therefore evaluated expression of ACE2 and TMPRSS2 as well as other novel molecular players of AKI and AKI-lung cross-talk in publicly available microarray datasets GSE6730 and GSE60088, which represented gene expression of lungs and kidneys in mouse models that resembled lung-kidney injury seen during SARS-CoV-2 infection.

\section{Results}

Expression of COVID-19 related genes ACE2 and TMPRSS2 was downregulated in lungs at early stages of injury. At a later stage, the expression of ACE2 decreased further, while expression of TMPRSS2 recovered. In kidneys, both genes were downregulated by AKI, but not by distant lung injury. We also identified 53 kidney genes upregulated by pneumonia and mechanical ventilation (PMV); and 254 lung genes upregulated by AKI, 9 genes of which were common to both organs. 3 of 9 genes were previously linked to kidney-lung cross-talk: Lcn2 (Fold Change (FC) Lung (L) $_{\text {I }}$ 18.6, FC Kidney 
$(\mathrm{K})=6.32)$, Socs3 $\left(\mathrm{FC}_{\mathrm{L}}=10.5, \mathrm{FC}_{\mathrm{K}}=10.4\right)$, Inhbb $\left(\mathrm{FC}_{\mathrm{L}}=6.20, \mathrm{FC}_{\mathrm{K}}=6.17\right)$. This finding validates the current approach and reveals new 6 candidates, including $\mathrm{Maff}\left(\mathrm{FC}_{\mathrm{L}}=7.21, \mathrm{FC}_{\mathrm{K}}=5.98\right)$.

\section{Conclusions}

Using our in-silico approach, we identified changes in COVID-19 related genes ACE2 and TMPRSS2 in traditional mouse models of AKI and lung cross-talk. We also found changes in the new candidate genes, which could be involved in the combined kidney-lung injury during COVID-19. 


\section{Background}

Publicly available genomics datasets have been growing drastically during the past decades. Although most of such datasets were initially generated to answer a pre-defined scientific question, the enormous amount of accumulated data makes genomics data re-purposing possible when new challenges such as COVID-19 arise and need to be rapidly dealt with. These data sets are also very useful to study when traditional wet-lab research is slowed down during the pandemic, and experimental models of COVID19 are still under development.

Clinical data for COVID-19 suggested that its mortality and morbidity can be linked not only to respiratory complications but also to other organs dysfunction [1]. Acute kidney injury (AKI) is now well established as a consequence of COVID-19. There is a strong association between AKI and dysfunction of extra-renal organs, and the more recently animal research has shown a significant causal effect of AKI on a distant organ dysfunction [2-6]. Despite frustrating outcomes, little is known about the potential pathophysiological interactions between the kidney and the extrarenal organs in critically ill patients, including COVID-19. There is a pressing need to study COVID-19 related genes and kidney-lung cross talk to discover novel diagnostic and therapeutic targets.

We previously used combined genomics data analysis of kidney and lung to discover new molecular pathways of kidney-lung cross talk [7], and demonstrated that inflammation is a major component of the initiation and exacerbation of AKI. To more closely relate our findings to COVID-19, we re-visited the global gene expression profiles of kidney in mice with pneumonia and mechanical ventilation $[8,9]$. These data set, which allowed us to focus on changes in two important COVID-19 related molecules that were previously ignored during studies of kidney-lung crosstalk. SARS-CoV-2 uses angiotensinconverting enzyme 2 (ACE2), to enter both kidney and lung, and is highly expressed in proximal tubule cells of the kidney [8]. Transmembrane protease serine 2 (TMPRSS2) is robustly expressed in the distal nephron [10] and facilitates the virus cell entry via ACE2. In addition to finding significant changes in 
expression of ACE2 and TMPRSS2 in these two models, we also identified novel candidates that could mediate cross-talk between the lung and kidney that could be pertinent to COVID-19.

\section{Methods}

Study aim.

This expression data re-purposing study aims to predict the genetic basis for cross-talk between SARSCoV-2 infected kidney and lung using publicly available data. Mouse models were selected based upon phenotypes similar to those in patients with COVID-19. Our hypothesis is that we will be able to identify gene candidates that might be related to the infection response and can be used to generate new hypotheses.

Two major COVID-19 related clinical conditions were selected: acute respiratory distress syndrome (ARDS) and acute kidney injury. The microarray Genomic Series GSE60088 and GSE6730 were downloaded from the Gene Expression Omnibus (GEO).

Brief description of gene expression series 60088: The S. aureus ( $\left.10^{7} \mathrm{cfu}\right)$ was deposited in mice oropharynx ( $n=5)$ and mice were intubated via tracheostomy with a 20-gauge blunt metal catheter. Intubated mice were connected to a MiniVent rodent ventilator and mechanically ventilated with a tidal volume of $10 \mathrm{~mL} / \mathrm{kg}$, a respiratory rate of 150 breaths per minute. Control mice $(\mathrm{n}=5)$ were maintained in their cages. After 6 hours the lungs and kidneys were collected for RNA isolation and analysis by Mouse Genome 4302.0 microarray chip (Affymetrix) for differentially expressed genes, GEO-build-in GEO2R tool was used. The groups were defined as follow: Control Lung Genomic Sample GSM1464822-GSM1464825, MV+SA Lung GSM1464826-GSM14648230. Control Kidney GSM1464839-GSM1464843, MV+SA Kidney GSM1464844-GSM1464848. Then results for all genes were downloaded.

Brief description of gene expression series 6730: Male 6-8-week-old mice (C57BL6/J), weighing approximately 25-30 grams were placed on a heating blanket and underwent midline laparotomy with 
isolation of bilateral renal pedicles. For mice assigned to experimental ischemia-reperfusion injury (IRI), a non-traumatic microvascular clamp was applied across both renal pedicles for 60 minutes. After the allotted ischemia time, the clamps were gently removed, the animals were administered $1 \mathrm{ml}$ of sterile saline intraperitoneally, and the incision was the closed in two layers with 4-0 silk suture. The animals were then allowed to recover with free access to food and water. Sham animals underwent the identical procedure without placement of the vascular clamps. At 6 or 36 hours following the experimental procedure, the mice were euthanized by exsanguination under pentobarbital anesthesia and lung tissues collected for the analysis. The total RNA was isolated and hybridized to a Mouse Genome 4302.0 microarray (Affymetrix).

Statistical analysis.

To identify differentially expressed genes, GEO-build-in GEO2R tool was used. The groups were defined as follow: Control 36h GSM155092- GSM155096, AKI 36h GSM155100- GSM155102. Control 6h GSM155086- GSM155088, AKI 6h GSM155089- GSM155091. Then results for all the genes were downloaded. The main SARS-CoV-2 targets: angiotensin-converting enzyme 2 (ACE2) and transmembrane protease, serine 2 (TMPRSS2) were selected for the detailed expression analysis.

\section{Results}

We identified changes in lungs and kidneys of COVID-19 related genes ACE2 and TMPRSS2 in pneumonia and mechanical ventilation (PMV) and ischemic AKI-induced lung dysfunction (Table 1). Expression of ACE2 in lungs was significantly downregulated after 6 hours of PMV, similar to downregulation in lungs at 6 hours after the induction of ischemic AKI. Lung ACE2 was further down regulated 36 hours after ischemic AKI. (Table 1). Expression of TMPRSS2 in lung was also significantly decreased after PMV, as it was 6 hours after ischemic AKI. However, at 36 hours of AKI lung TMPRSS2 was similar to the baseline. 
In kidneys ACE2 sharply decreased at 6 hours after AKI and was further downregulated 36 hours after the induction of ischemic AKI. However, kidney ACE2 was unaffected by PMV. Kidney TMPRSS2 was unaffected in PMV model, but decreased 6 hours after ischemic AKI. (Table 1). We then explored other molecular targets in both organs by filtering for known genes with P-value $<0.01$ and fold change $(\mathrm{FC})>4$. This approach identified 53 genes upregulated in kidneys from mice with PMV; and 254 genes upregulated in lungs from mice with AKI (Figure 1). Cross-referencing of these candidates identified 9 common genes (Table 2). Three of them Lcn2, Socs3, and Inhbb had already been associated with kidney-lung cross-talk [7], which validate by our current approach. We also identify 6 novel molecular targets for kidney lung-cross talk.

\section{Discussion}

Serious COVID-19 disease is usually characterized by lung injury and often associated with acute kidney injury. There has been mechanistic study of combined AKI and ARDS in animal models, but not related to COVID-19. Prior studies focusing on mechanisms of kidney-lung cross talk did not examine COVID-19 specific genes. Publicly available databases can be re-analyzed while labs are slowed down and animal models of COVID-19 are being developed. We used this approach and found unexpected decreases in key SARS-CoV2 molecules ACE2 and TMPRSS2 in two models of kidney lung cross talk, pneumonia and mechanical ventilation and AKI. We also validated our re-analysis by identifying 3 previously described genes that were associated with kidney-lung cross talk, and identified 6 novel targets.

ACE2 and TMPRSS2 are important for SARS-CoV2 entry into lungs and kidneys. It is believed that levels of these molecules predispose to entry, infection and disease development, and could explain why some get more serious injury while others do not [11]. There are also "second" hits that can alter patient outcome, such as bacterial pneumonia, mechanical ventilation induced injury, and AKI. We examined expression of these two molecules in two traditional published models and expected an 
increase in mRNA expression of the receptors. Unexpectedly, both ACE2 and TMPRSS2 decreased in lung and kidney during PMV and AKI, though with different intensities and kinetics, while other genes increased. The meaning of this is unknown and could be tested in the future studies when experimental models of COVID-19 are better developed. However, one can speculate that a "second hit" could make it more difficult for SARS-CoV-2 to enter lung and kidney cells and might be protective. Based on the level of gene expression in Table 2, we directly extrapolated the gene expression values to receptor expression on the surface of a cell and build our protective model against SARS-CoV-2 (Figure 2).

While focusing on the important COVID-19 targets ACE-2 and TMPRSS2 molecules, we re-analyzed these data sets and confirmed that our approach was valid when we identified 3 previously known candidates of lung-kidney cross talk during AKI: lipocalin 2 (NGAL), suppressor of cytokine signaling 3 (member of STAT family), and inhibin beta B. NGAL is a bio-marker of AKI but also has important role in iron metabolism, epithelial cell and immune cell functions. Socs3 in stressed proximal tubules plays an important role during AKI by inhibition of reparative proliferation [12]. Inhibin beta B is a member of the transforming growth factor $\beta$ (TGF- $\beta$ ) superfamily. It has been reported that this gene is significantly up-regulated in renal endothelial cells from male mice with IRI [13]. We also identified 6 new candidate kidney-lung cross talk genes. We found Maff gene particularly interesting. This gene is abundantly expressed in kidney and its up-regulation has been linked to deleterious effects on podocytes [14]. Maff also binds the oxytocin receptor promoter [15], which could contribute to sex differences in disease outcome - seen in COVID-19 [16].

\section{Conclusions}

Study of ACE-2 and TMPRSS2 molecular pathways during ARDS and AKI are important both in patients and in experimental models to understand better the pathogenesis of COVID-19 and fashion improved therapies. They may also interplay with other key genes during kidney-lung cross talk in the critically ill patient. Presented here analytical method of re-purposing available genomics data allowed 
us to generate COVID-19 related hypothesis, which can be studied and validated in increasingly established COVID models. Moreover, this method is generalizable and can be applied to different studies.

\section{Abbreviations}

ACE-2: $\quad$ Angiotensin-converting enzyme 2

AKI: $\quad$ Acute kidney injury

CoV: $\quad$ Corona virus

COVID: $\quad$ Corona virus disease

FC: $\quad$ Fold change

GEO: $\quad$ Gene Expression Omnibus

IRI: $\quad$ Ischemia reperfusion injury

Maff: $\quad$ musculoaponeurotic fibrosarcoma oncogene family $\mathrm{F}$

NGAL: Neutrophil gelatinase-associated lipocalin

PMV: $\quad$ Pneumonia and mechanical ventilation

SARS: $\quad$ Severe acute respiratory syndrome

Socs3: $\quad$ Suppressor of cytokine signaling 3

TGF- $\beta$ : $\quad$ Transforming growth factor $\beta$

TMPRSS2: Transmembrane protease serine 2

\section{Declarations}

Ethics approval and consent to participate:

Given that the data for this study was obtained from publicly available repository, the authors do not have a detailed ethics approval information. Therefore, ethical statements are reproduced from the data submission page of GEO and pertinent manuscripts. 
GSE6730: All procedures were approved by the Johns Hopkins Animal Care and Use Committee, and were consistent with the National Institutes of Health (NIH) Guide for the Care and Use of Laboratory Animals [7].

GSE60088: The Office of Animal Welfare at the University of Washington approved all experiments [9].

Consent for publication:

Both authors are consenting for the publication.

Availability of data and materials:

The data supporting the results reported in this manuscript can be found at GEO:

https://www.ncbi.nlm.nih.gov/geo/query/acc.cgi?acc=GSE60088

https://www.ncbi.nlm.nih.gov/geo/query/acc.cgi?acc=GSE6730

Competing interests:

The authors have no competing interest.

Funding:

This in silico study was conducted outside of any funding agencies.

Authors' contributions:

HR proposed and guided the study, revised the manuscript.

DNG participated in protocol editing and conduct the study.

All authors have approved the final manuscript and certify that this manuscript represents valid work and has not been previously published. 
Acknowledgments:

The study investigators thank the owners of the raw expression data, who made it available for other investigators.

Authors' information (optional):

Dmitry N. Grigoryev MD, PhD, Sr. Bioinformatician, Center for Translational Data Science, University of Chicago Division of Biological Sciences, Chicago, IL, USA

Hamid Rabb MD, Director, Kidney Transplant Program, Johns Hopkins Medicine, Baltimore, MD 


\section{References}

1. Kow CS, Hasan SS: Possible protective effect of renin-angiotensin system inhibitors in COVID-19 induced acute kidney injury. J Am Soc Nephrol 2020; 31(8):1917-1918.

2. Hoke TS, Douglas IS, Klein CL, He Z, Fang W, Thurman JM, et al. Acute renal failure after bilateral nephrectomy is associated with cytokine-mediated pulmonary injury. J Am Soc Nephrol 2007; 18(1):155-164.

3. Hassoun H, Grigoryev DN, Lie M, Liu M, Cheadle C Tuder RM, Rabb H. Ischemic acute kidney injury induces a distant organ functional and genomic response distinguishable from bilateral nephrectomy. 2007; Am J Physiol Renal Physiol 293(1):F30-40.

4. Kelly KJ: Distant effects of experimental renal ischemia/reperfusion injury. J Am Soc Nephrol 2003; 14(6):1549-1558.

5. Rabb H, Wang Z, Postler G, Soleimani M: Possible molecular basis for changes in potassium handling in acute renal failure. Am J Kidney Dis 2000; 35(5):871-877.

6. Zarbock A, Schmolke M, Spieker T, Jurk K, Van Aken H, Singbartl K. Acute uremia but not renal inflammation attenuates aseptic acute lung injury: a critical role for uremic neutrophils. J Am Soc Nephrol 2006; 17(11):3124-3131.

7. Grigoryev DN, Liu M, Hassoun HT, Cheadle C, Barnes KC, Rabb H. The local and systemic inflammatory transcriptome after acute kidney injury. J Am Soc Nephrol 2008; 19(3):547-558.

8. Gu C, Qiao W, Wang L, Li M, Song K. Identification of genes and pathways associated with multiple organ dysfunction syndrome by microarray analysis. Mol Med Rep 2018; 18(1):31-40.

9. Gharib SA, Mar D, Bomsztyk K, Denisenko O, Dhanireddy S, Liles WC, Altemeier WA. System-wide mapping of activated circuitry in experimental systemic inflammatory response syndrome. Shock 2016; 45(2):148-156.

10. Ni W, Yang X, Yang D, Bao J, Li R, Xiao Y, et al. Role of angiotensin-converting enzyme 2 (ACE2) in COVID-19. Crit Care 2020; 24(1):422.

11. Batlle D, Soler MJ, Sparks MA, Hiremath S, South AM, Welling PA, Swaminathan S. acute kidney injury in COVID-19: Emerging evidence of a distinct pathophysiology. J Am Soc Nephrol 2020; 31(7):1380-1383.

12. Susnik N, Sörensen-Zender I, Rong S, Vietinghoff S, Lu X, Rubera I, et al. Ablation of proximal tubular suppressor of cytokine signaling 3 enhances tubular cell cycling and modifies macrophage phenotype during acute kidney injury. Kidney Int. 2014; 85(6):1357-68

13. Vinas JL, Porter CJ, Douvris A, Spence M, Gutsol A, Zimpelmann JA, et al. Sex diversity in proximal tubule and endothelial gene expression in mice with ischemic acute kidney injury. Clin Sci 2020; 134 1887-1909

14. Okabe M, Motojima M, Miyazaki Y, Pastan I, Yokoo T, Matsusaka T. Global polysome analysis of normal and injured podocytes. Am J Physiol Renal Physiol 2019; 316: F241-F252

15. Kimura T, Ivell R, Rust W, Mizumoto Y, Ogita K, Kusui C, et al. Molecular cloning of a human MafF homologue, which specifically binds to the oxytocin receptor gene in term myometrium. Biochem Biophys Res Commun 1999; 264(1):86-92.

16. Jin JM, Bai P, He W, Wu F, Liu XF, Han DM, et al. Gender Differences in Patients With COVID-19: Focus on Severity and Mortality. Front Pub Health 2020; 8:152. 
Table 1. Response of ACE2 and TMPRSS2 to injurious stimuli in kidney and lung.

\begin{tabular}{c|lccc|cc} 
& & \multicolumn{2}{c}{ ACE2 } & \multicolumn{2}{c}{ TMPRSS2 } \\
\hline \multicolumn{1}{c}{ Organ } & Condition & FC & P-value & FC & P-value \\
\hline \multirow{4}{*}{ LUNG } & PMV & -1.71 & 0.00037 & -1.69 & 0.00002 \\
& AKI 6hrs & -1.86 & 0.00116 & -1.43 & 0.00022 \\
& AKI 36hrs & -2.56 & 0.00062 & -1.27 & NS \\
\hline \multirow{3}{*}{ KIDNEY } & PMV & 1.05 & NS & 1.17 & NS \\
& AKI 6hrs* & -2.44 & 0.00108 & -1.76 & 0.01224 \\
\cline { 2 - 6 } & AKI 36hrs* & -3.27 & 0.00033 & 1.03 & NS \\
\hline
\end{tabular}

* - the data was extracted from supplemental materials Grigoryev et al [7]

AKI - acute kidney injury

PMV - pneumonia and mechanical ventilation

$\mathrm{FC}$ - fold change

Table 2. Gene candidates distantly upregulated in kidney or lung by injury to distant organ.

\begin{tabular}{llcccc}
\hline \multirow{2}{*}{ Gene } & \multirow{2}{*}{ Symbol } & \multicolumn{2}{c}{ lung during AKI } & \multicolumn{2}{c}{ kidney in PMV } \\
\cline { 3 - 7 } & & FC & P value & FC & P value \\
\hline Lipocalin 2 & Lcn2 & $\mathbf{1 8 . 5 8}$ & 0.00020 & $\mathbf{6 . 3 2}$ & 0.00720 \\
\hline Suppressor of cytokine signaling 3 & Socs3 & $\mathbf{1 0 . 5 2}$ & 0.00018 & $\mathbf{1 0 . 3 6}$ & 0.00058 \\
\hline $\begin{array}{l}\text { V-maf musculoaponeurotic fibrosarcoma oncogene } \\
\text { family F }\end{array}$ & \multirow{2}{*}{ Maff } & & & & \\
\hline Jun B proto-oncogene & Junb & $\mathbf{6 . 2 1}$ & 0.00221 & $\mathbf{5 . 9 8}$ & 0.00091 \\
\hline Inhibin beta-B & Inhbb & $\mathbf{6 . 2 0}$ & 0.00023 & $\mathbf{5 . 3 7}$ & 0.00194 \\
\hline $\begin{array}{l}\text { Tumor necrosis factor receptor superfamily, member } \\
\text { 12a }\end{array}$ & \multirow{2}{*}{ Tnfrsf12a } & $\mathbf{6 . 0 9}$ & 0.00063 & $\mathbf{6 . 1 7}$ & 0.00102 \\
\hline Cholesterol 25-hydroxylase & Ch25h & $\mathbf{6 . 0 4}$ & 0.00040 & $\mathbf{4 . 3 0}$ & 0.00081 \\
\hline Metallothionein 2 & Mt2 & $\mathbf{5 . 2 9}$ & 0.000078 & $\mathbf{7 . 0 9}$ & 0.00003 \\
\hline 3-hydroxy-3-methylglutaryl-Coenzyme A synthase 2 & Hmgcs2 & $\mathbf{4 . 4 4}$ & 0.00877 & $\mathbf{5 . 3 1}$ & 0.00044 \\
\hline
\end{tabular}

AKI - acute kidney injury

PMV - pneumonia and mechanical ventilation

FC - fold change 


\section{Figure legends}

\section{Figure 1. Theoretical model of kidney-lung interaction during COVID-19.}

We postulate that SARS-CoV2 enters lung and perhaps kidney using TMPRSS2 genes for priming to ACE-2 and ACE-2 itself as a main entry point. Second hits, like ventilation injury and AKI (local tissue inflammation) can alter the expression of these two genes and change response to further infection. In addition, key pathways are altered in lung and kidney that in turn modify survival and tissue injury/repair. In addition to ACE-2 and TMPRSS2, additional important genes could be involved in lung-kidney cross talk in the critically ill patient (the depicted downregulation values are taken from Table 1 and represent genomic effects at 6 hours after injury). Changes in 9 genes were significant during experimental PMV and AKI; and were common to both organs during injury, of which three were previously described as AKI genes (Lcn2, Socs3, and Inhbb); other six genes were novel (Mt2, Maff, Junb, Hmgcs2, Tnfrsf12a and Ch25h).

\section{Figure 2. Protective model of a "second hit" during distant organ injury.}

The proposed model demonstrates that with increased time after the initial injury it becomes more difficult for SARS-CoV-2 to enter the injured lung or kidney cell. At 6 hours after the injury the both targets of SARS-CoV-2 (ACE-2 and TMPRSS2) are down regulated. At 36 hours after the injury, while the expression of TMPRSS2 is recovered, the expression of ACE-2 is down regulated even more than at 6 hours. 


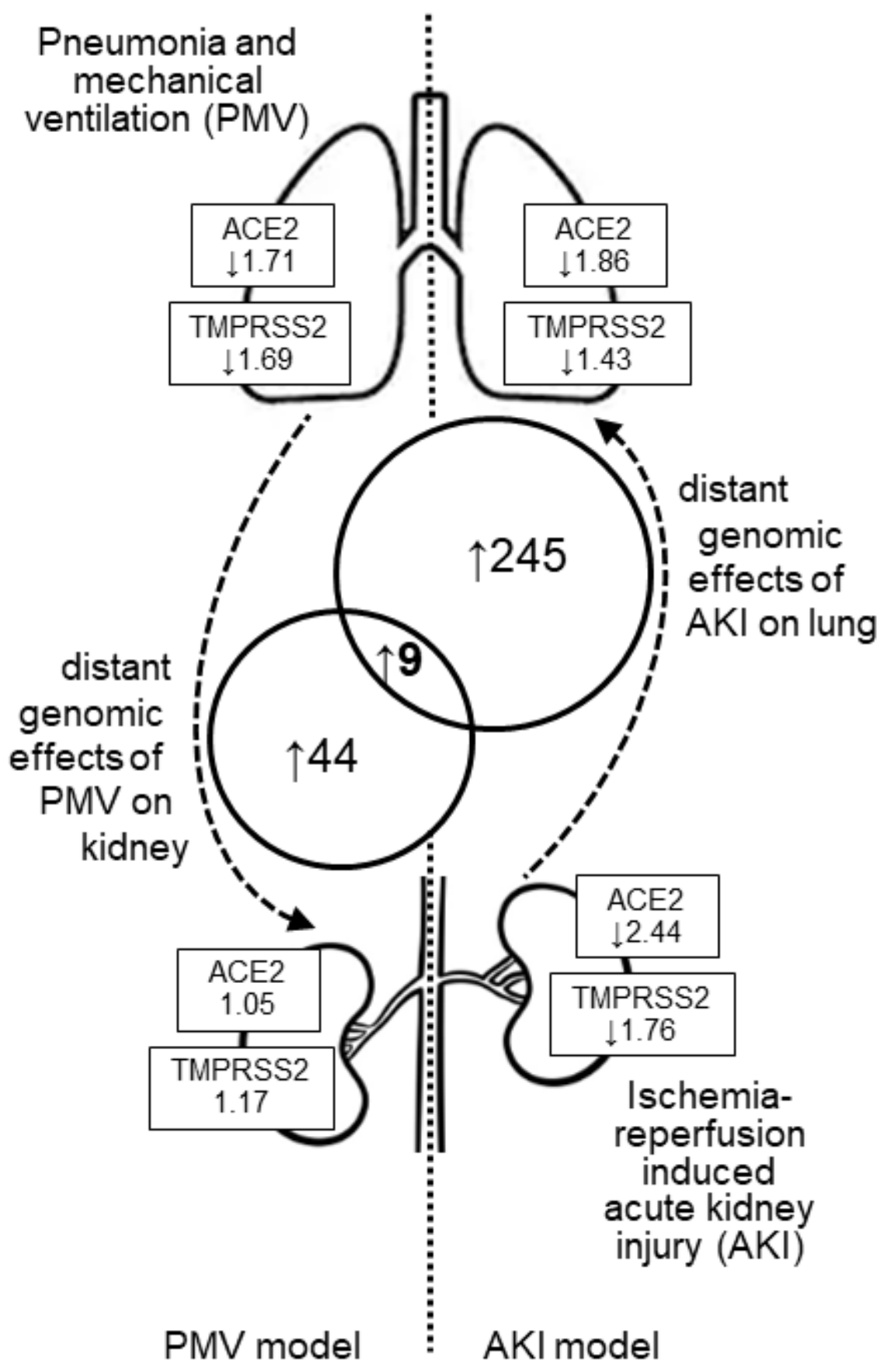

$\uparrow \downarrow$ - significant updown- regulation

\section{Figure 1}

Theoretical model of kidney-lung interaction during COVID-19. We postulate that SARS-CoV2 enters lung and perhaps kidney using TMPRSS2 genes for priming to ACE-2 and ACE-2 itself as a main entry point. Second hits, like ventilation injury and AKI (local tissue inflammation) can alter the expression of these two genes and change response to further infection. In addition, key pathways are altered in lung and kidney that in turn modify survival and tissue injury/repair. In addition to ACE-2 and TMPRSS2, additional 
important genes could be involved in lung-kidney cross talk in the critically ill patient (the depicted downregulation values are taken from Table 1 and represent genomic effects at 6 hours after injury). Changes in 9 genes were significant during experimental PMV and AKl; and were common to both organs during injury, of which three were previously described as AKI genes (Lcn2, Socs3, and Inhbb); other six genes were novel (Mt2, Maff, Junb, Hmgcs2, Tnfrsf12a and Ch25h).

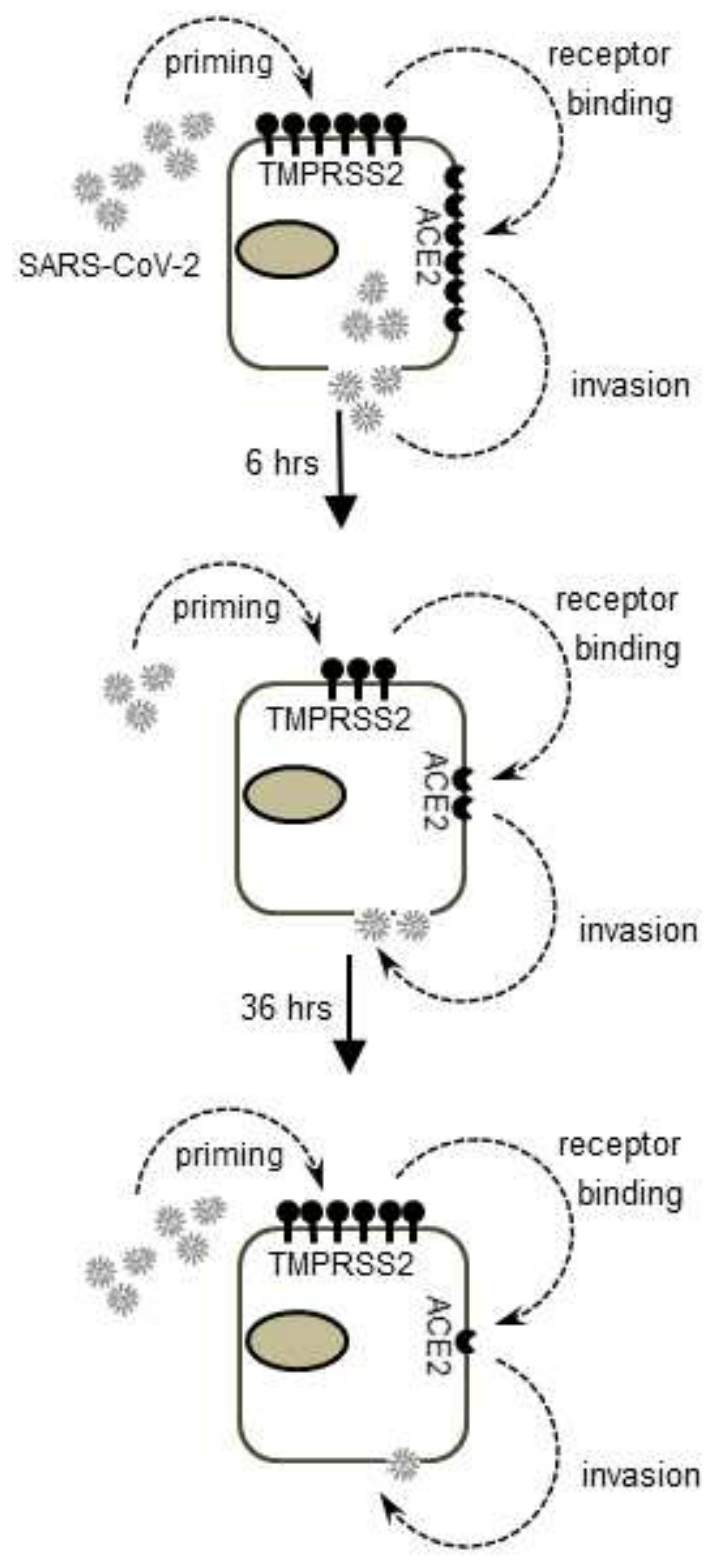

Figure 2

Protective model of a "second hit" during distant organ injury. The proposed model demonstrates that with increased time after the initial injury it becomes more difficult for SARS-CoV-2 to enter the injured lung or kidney cell. At 6 hours after the injury the both targets of SARS-CoV-2 (ACE-2 and TMPRSS2) are down regulated. At 36 hours after the injury, while the expression of TMPRSS2 is recovered, the expression of ACE- 2 is down regulated even more than at 6 hours. 